\title{
Natalizumab exerts a suppressive effect on surrogates of B cell function in blood and CSF
}

\author{
Clemens Warnke, Mark Stettner, Vera Lehmensiek, Thomas Dehmel, Anne K Mausberg, Gloria \\ von Geldern, Ralf Gold, Tania Kümpfel, Reinhard Hohlfeld, Mathias Mäurer, Martin Stangel, \\ Vera Straeten, Volker Limmroth, Thomas Weber, Christoph Kleinschnitz, Mike P Wattjes, \\ Anders Svenningsson, Tomas Olsson, Hans-Peter Hartung, Derik Hermsen, Hayrettin Tumani, \\ Ortwin Adams and Bernd C Kieseier
}

\section{Abstract}

Background: Natalizumab for multiple sclerosis (MS) increases the risk of progressive multifocal leukoencephalopathy (PML).

Objective: We aimed to assess the effect of natalizumab on cellular composition and functional B cell parameters including patients with natalizumab-associated PML $(n=37)$.

Methods: Cellular composition by flow cytometry, levels of immunoglobulin (Ig)G/IgM by immunonephelometry, and oligoclonal bands by isoelectric focusing were studied in blood and cerebrospinal fluid.

Results: In MS patients treated with natalizumab without PML $(n=59)$ the proportion of CD19+ B cells was higher in blood, but lower in cerebrospinal fluid compared with MS patients not treated with natalizumab $(n=17)$. The CD4/CD8-ratio in cerebrospinal fluid was lower, and IgG and IgM levels as well as the IgG index dropped in longitudinal samples during natalizumab therapy. Oligoclonal bands persisted, but the total amount of the intrathecally produced $\mathrm{IgG}$ fraction, and the polyclonal intrathecal IgG reactivity to measles, rubella, and zoster declined. At the time of diagnosis of PML patients with natalizumabassociated PML had low total IgG levels in blood and cerebrospinal fluid.

Conclusions: Natalizumab impacts B and T cell distribution and exerts an inhibitory effect on surrogates of B cell function in periphery and in cerebrospinal fluid, potentially contributing to the increased risk of developing PML.

Keywords: Progressive multifocal leukoencephalopathy, JC virus, natalizumab, Tysabri, multiple sclerosis, disease-modifying therapies, immunosuppression

Date received: 15 June 2014; revised: 5 August 2014; 7 September 2014; accepted: 8 September 2014

\section{Introduction}

Natalizumab, a monoclonal antibody against the $\alpha 4$ integrin, is approved for relapsing-remitting multiple sclerosis (MS) and prevents the transmigration of activated $\mathrm{T}$ cells across the blood-brain barrier into the brain tissue. ${ }^{1}$ Reported changes during natalizumab therapy are an inversion of the $\mathrm{CD} 4 / \mathrm{CD} 8$ ratio in cerebrospinal fluid (CSF), a reduction of antigen-presenting cells in the perivascular spaces, ${ }^{2,3}$ and an effect on the immune repertoire of $\mathrm{T}$ cells in blood and $\mathrm{CSF}^{4}$

However, the most prominent observation in the peripheral blood cell count of patients treated with natalizumab is an increase in CD34+ hematopoietic progenitors $^{5}$ as well as of circulating pre-B and $\mathrm{B}$ cells. ${ }^{6,7}$ This increase can be explained by an impaired homing, ${ }^{7,8}$ and/or a diminished adhesion of these cells to the natural niches. ${ }^{9}$ Adhesion signalling at natural niches provides pro-survival stimuli in particular to antibody-producing cells. ${ }^{10}$ Inhibitory effects on B-cell function might be an important part of the mode of action of natalizumab, and potentially are also linked to the increased risk of developing progressive multifocal leukoencephalopathy (PML) or herpes virus complications. ${ }^{11-13}$ Recent publications suggest that natalizumab might alter the humoral
Multiple Sclerosis Journal 2015, Vol. 21(8) 1036-1044 DOI: $10.1177 /$ 1352458514556296

(C) The Author(s), 2015. Reprints and permissions: http://www.sagepub.co.uk/ journalsPermissions.nav
Correspondence to:

Clemens Warnke

Department of Neurology,

Moorenstraße 5, 40225

Duesseldorf, Germany.

clemens.warnke@med.uniduesseldorf.de

Clemens Warnke Mark Stettner

Thomas Dehmel

Anne K Mausberg

Hans-Peter Hartung Bernd C Kieseier

Department of Neurology, Medical Faculty, Heinrich-Heine-University, Duesseldorf, Germany

Vera Lehmensiek

Hayrettin Tumani Department of Neurology, University of Ulm, Ulm, Germany

Gloria von Geldern

National Institute of

Neurological Disorders and Stroke, NIH, Bethesda, MD USA

Ralf Gold

Department of Neurology, Ruhr University, Bochum,

Germany

Tania Kümpfel Reinhard Hohlfeld Institute of Clinical

Neuroimmunology, Ludwig Maximilian University and Munich Cluster for Systems

Neurology (SyNergy), Munich, Germany

Mathias Mäurer Department of Neurology, Caritas Hospital, Bad Mergentheim, Germany

Martin Stangel Clinical Neuroimmunology and Neurochemistry, Department of Neurology, Hanover Medical School, Hanover, Germany

Vera Straeten Department of Neurology, Johannes Wesling Hospital Minden, Minden, Germany

Volker Limmroth

Department of Neurology, 
immune response toward $\mathrm{JC}$ virus (JCV) and varicella zoster virus (VZV), ${ }^{14}$ decreases serum immunoglobulin (Ig)M and IgG levels, ${ }^{15}$ and potentially also surrogates of intrathecal antibody synthesis. ${ }^{16,17}$

The aim of this study was to assess the effects of therapy with natalizumab in MS patients on the cellular composition and on B cell functional parameters in blood and CSF including patients with natalizumabassociated PML.

\section{Materials and methods}

\section{Patients}

The study was performed as part of the German pharmacovigilance study for natalizumab at the Department for Neurology, Heinrich-Heine University Duesseldorf, Germany. The study included 59 patients with relapsing-remitting MS treated with natalizumab for more than 18 months, and 17 age- and gender-matched patients with relapsing forms of MS not treated with natalizumab ${ }^{4}$ who did not receive any disease-modifying therapy within the preceding 2 months of study inclusion. In addition, IgG levels in blood and CSF were available from 37 patients with natalizumab-associated PML, of which 20 were first sampled at time of diagnosis as defined by a first positive PCR for JCV DNA in CSF. The CSF anti-JCV IgG antibody index in this cohort of PML patients has been recently published..$^{18}$ The study was approved by the local institutional review board (protocol number 3315). All non-PML MS patients gave written informed consent to the scientific use of their blood and CSF samples and data. The local Ethics committee waved the requirement for written informed consent for the use of anonymized data of patients with PML available at the Institute for Virology.

\section{Flow cytometric analysis, immunonephelometry}

and isoelectric focusing

The cellular composition of blood and CSF was assessed by fluorescence-activated cell sorting (FACS), using fluorescence-labelled antibodies against CD14, CD19, CD3, CD4 and CD8 (all BD Bioscience, according to the manufacturer's protocol). Fresh ethylenediaminetetraacetic acid (EDTA) blood and CSF samples were used for analysis performed immediately after sampling. IgG, IgM and albumin were determined as published, ${ }^{18}$ and the $\operatorname{IgG}$ and the $\operatorname{IgM}$ index, and the locally produced absolute IgG fraction (IgG-loc) were calculated. Detection of oligoclonal bands (OCBs) was performed by isoelectric focusing (IEF) on polyacrylamide gels followed by immunoblotting using an
IgG-specific antibody staining. Paired CSF and serum adjusted for protein concentrations were applied in the same assay as described earlier, ${ }^{19}$ and OCBs were classified in five patterns as published. ${ }^{20}$ In addition, the polyspecific intrathecal antibody responses to measles, rubella and zoster were studied by enzyme-linked immunosorbent assay (ELISA) (Euroimmun, Lübeck, Germany), and the antibody specificity index was calculated as published. ${ }^{21}$

\section{Statistical analysis}

Graph Pad Prism version 5.0 (GraphPad Software, San Diego, USA) was used for statistical analysis. 95\% confidence intervals (CI) for proportions were calculated using the modified Wald method; for nonparametric measures the Mann-Whitney, or the Kurskal-Wallis test including Dunn's test to correct for multiple comparisons was applied. For longitudinal studies, the Wilcoxon matched pairs test was used; $p$-values $<0.05$ were considered significant.

\section{Results}

\section{Patients' characteristics}

MS patients treated with natalizumab and non-natalizumab-treated MS patients were age- and gendermatched. ${ }^{4}$ Patients with natalizumab-associated $\mathrm{PML}^{18}$ were older $(p=0.003)$, and had longer duration of therapy with natalizumab $(p<0.0001)$ compared with MS patients treated with natalizumab without PML (Table 1). In 8 of the 59 of the patients during long-term therapy with natalizumab, a CSF-serum pair obtained prior to natalizumab therapy was available, allowing us to studying the intra-individual changes following natalizumab treatment initiation (median time between sampling: 30.5 months, range 21-55; no disease-modifying therapy within 4 weeks prior to natalizumab).

\section{The cellular composition of peripheral blood and CSF during therapy with natalizumab}

Compared with MS patients not treated with natalizumab, natalizumab-treated patients had a higher relative number of $\mathrm{CD} 19+\mathrm{B}$ cells in the peripheral venous blood (median: $16.4 \%$ vs. $6.7 \% ; p<0.0001$ ), and a lower proportion of CD19+ B cells in CSF (median: $0.4 \%$ vs. $2.4 \%$; $p<0.007$ ). The proportions of $\mathrm{CD} 14+, \mathrm{CD} 3+$ cells and the CD4/CD8 ratio in blood did not significantly differ between MS patients treated or not treated with natalizumab. The CD4/ CD8 ratio in CSF was found to be inversed (median: 0.66 vs. $4.47, p<0.0001),{ }^{4}$ and the relative number of
Merheim Hospital, Cologne, Germany

Thomas Weber

Department of Neurology, Marienhospital Hamburg, Hamburg, Germany

Christoph Kleinschnitz Department of Neurology, University Hospital Wuerzburg, Wuerzburg, Germany

Mike P Wattjes MS Center Amsterdam and Department of Radiology, Nuclear Medicine \& PET Research, VU University

Medical Center, Amsterdam, the Netherlands

Anders Svenningsson

Department of Pharmacology and Clinical Neuroscience,

Umea University Hospital,

Umea, Sweden

Tomas Olsson

Department of Neurology,

Center for Molecular

Medicine, Karolinska

Institutet, Stockholm,

Sweden

Derik Hermsen

Institute for Clinical Chemistry and Laboratory

Diagnostics, University

Hospital Duesseldorf,

Germany

Ortwin Adams

Institute for Virology, Medical Faculty,

Heinrich-Heine-University, Duesseldorf, Germany 
Table 1. Patient characteristics.

\begin{tabular}{|c|c|c|c|c|c|c|}
\hline & Total $n$ & $\begin{array}{l}\text { Age [years] } \\
\text { median (range) }\end{array}$ & $\begin{array}{l}\text { Sex } n \text { female } \\
(\%)\end{array}$ & $\begin{array}{l}\text { Treatment duration } \\
\text { [months] median (range) }\end{array}$ & $\begin{array}{l}\text { EDSS median } \\
\text { (range) }\end{array}$ & $\begin{array}{l}\text { CSF }[\text { cells } / \mu \mathbf{l}] \\
\text { median (range) }\end{array}$ \\
\hline MS $^{6 *}$ & 17 & $41.5(22-70)$ & $11(65)$ & NA & $3.0(1-5.5)$ & $3(1-31)$ \\
\hline NAT $^{6}$ & 59 & $40(23-60)$ & $41(69)$ & $24(19-50)$ & $2.5(0-6.5)$ & $1(1-6)$ \\
\hline NAT-PML ${ }^{24}$ & 37 & $45(28-63)$ & $25(68)$ & $44(19-75)$ & NA & NA \\
\hline
\end{tabular}

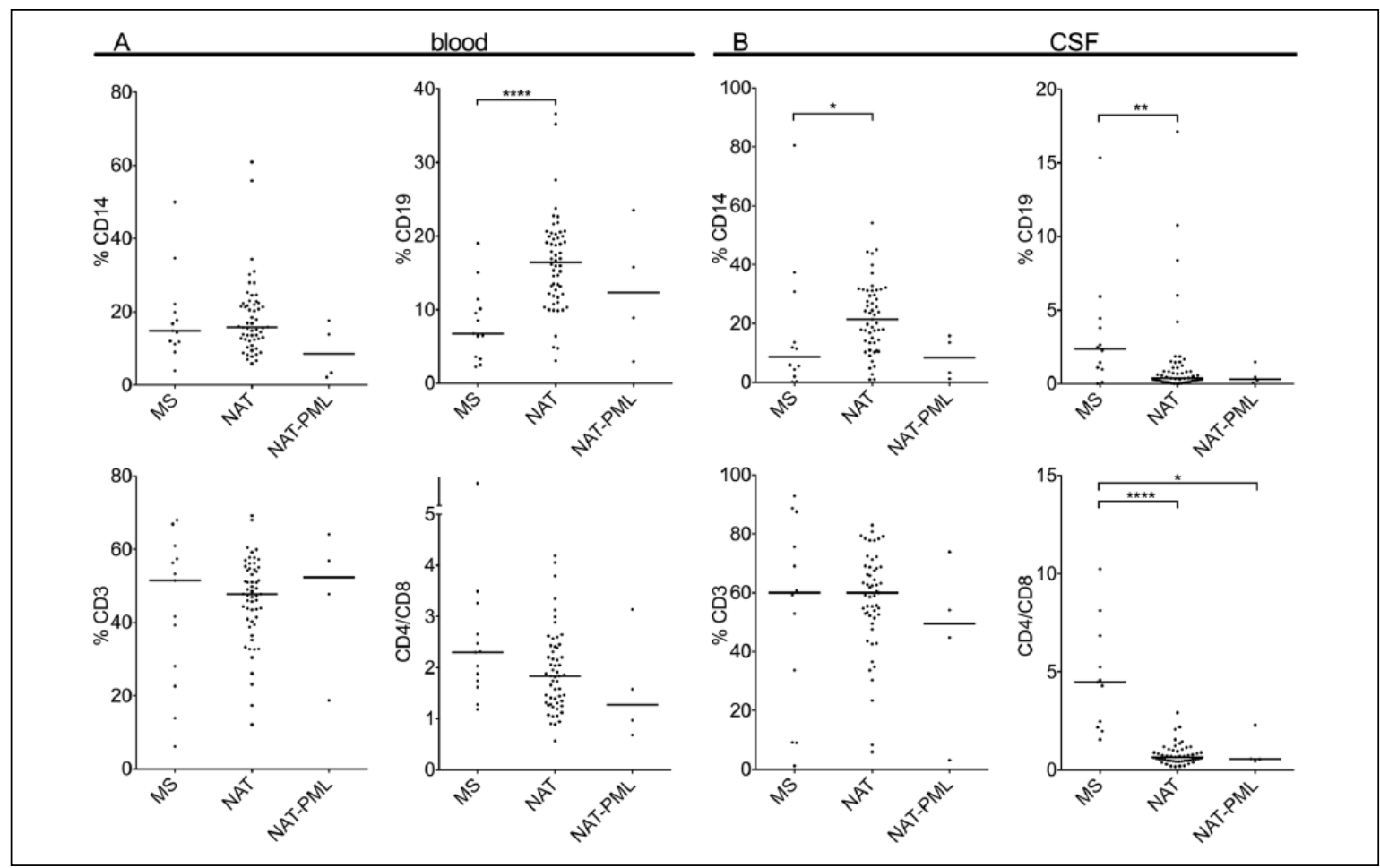

Figure 1. Cellular composition of blood (A) and cerebrospinal fluid (CSF; B) as assessed by FACS analysis. The relative number of $\mathrm{CD} 14+, \mathrm{CD} 19+, \mathrm{CD} 3+$ cells and the $\mathrm{CD} 4 / \mathrm{CD} 8$ ratio are shown. Bars represent the median. Statistics: Mann-Whitney. MS: patients with MS not treated with natalizumab; NAT: patients with MS treated with natalizumab; NAT-PML: patients with MS treated with natalizumab who developed PML.

CD14+ cells to be increased in CSF (median: $21.4 \%$ vs. $8.7 \% ; p=0.033$, Figure 1$)$.

In the limited number of PML patients $(n=4)$ with fresh EDTA and CSF cells available, CD19 proportions in CSF were found to be low (median: $0.33 \%$; $p=0.09$ for the comparison to non-natalizumab-treated MS patients), as well as the CD4/CD8 ratio (median: $0.57^{4} ; p=0.01$ for the comparison to non-natalizumabtreated MS patients) (Figure 1).

\section{IgG and IgM levels in blood and CSF during therapy with natalizumab}

IgG levels in patients with natalizumab-associated PML at the time of diagnosis of PML were lower compared with non-natalizumab-treated MS patients in blood (median: $7.77 \mathrm{~g} / 1$ vs. $9.66 \mathrm{~g} / \mathrm{l} ; p<0.001$ ) and CSF (median $18.40 \mathrm{mg} / \mathrm{l}$ vs. $26.35 \mathrm{mg} / \mathrm{l} ; p=0.011$ ). We noted a similar trend for natalizumab-treated MS patients without PML ( $p=0.08$ in blood and CSF); no correlation between treatment duration with natalizumab and IgG levels in blood (spearman $r: 0.15$ (95\% CI: -0.17-0.44)) or CSF (spearman $r: 0.13$ (95\% CI: -0.19-0.43)) was observed. In samples obtained from patients with natalizumab-associated PML during the course of PML (following plasma exchange (PLEX)) the IgG index was found to be increased. This observation appeared more prominent for samples taken 4 weeks after PLEX initiation or later. IgM levels were lower in blood (median: $0.63 \mathrm{~g} / 1$ vs. 1.40 $\mathrm{g} / \mathrm{l} ; p<0.001$ ) and CSF (median: $0.20 \mathrm{mg} / \mathrm{l}$ vs. 0.70 
$\mathrm{mg} / 1 ; p<0.0001)$ of patients treated with natalizumab compared with non-natalizumab-treated MS patients (Figure 2).

In longitudinal samples obtained before and during therapy with natalizumab, a decline in total $\operatorname{IgG}$ in blood (median of differences $-1.66 \mathrm{~g} / \mathrm{l} ; p=0.008$ ) and CSF (median of differences $-12.1 \mathrm{mg} / 1 ; p=0.008$ ), and total $\mathrm{IgM}$ in blood (median of differences $-0.48 \mathrm{~g} / \mathrm{l}$; $p=0.008$ ) and CSF (median of differences $-0.20 \mathrm{mg} / \mathrm{l}$; $p=0.008$ ) was noted (Figure 3 ).

\section{The intrathecally produced $\operatorname{Ig} G$ fraction during therapy with natalizumab}

The IgG index (median of differences $-0.18, p=0.031$ ) as a relative measure of the intrathecally produced IgG fraction declined in longitudinal samples collected before and during therapy with natalizumab (Figure 3). OCBs were detected in 49 out of 52 patients (94.2\%; 95\% CI: 83.7-98.6\%) during therapy with natalizumab. In all six patients with sufficient material for longitudinal re-assessment OCBs remained detectable, but the OCB intensity declined (Figure 4(A)). In line with this observation, the absolute concentration of the locally produced IgG fraction $(\mathrm{IgG}-\mathrm{loc})$ declined in four of these six patients. This was associated with a decline in antibody index to measles, rubella and zoster in three of the four patients with quantifiable intrathecal IgG synthesis (Figure 4(B)).

\section{Conclusions}

We here report that natalizumab exerts a suppressive effect on various different functional parameters of antibody synthesis. Specifically, we noted a decrease of IgG and IgM levels in blood and CSF of longitudinal samples, which is in line with an earlier report on lowered IgG and IgM levels in blood only. ${ }^{15}$ Partially conflicting findings in the literature on persistency of OCBs during natalizumab therapy are likely to be explained by differences in study design and methodology. While Harrer et al. analysed data aggregated from different laboratories and reported on disappearance of OCBs in $16 \%$ of individuals, ${ }^{17}$ Mancuso and colleagues performed a smaller longitudinal singlecentre study, noting complete disappearance of OCBs in as many as $55 \%$ of patients. ${ }^{16}$ In our mono-centric approach, OCBs remained detectable in the majority of our cross-sectionally studied patients (94\%), and in all six individuals with samples obtained before and during therapy with natalizumab. We confirmed longitudinal OCB persistency in a reference laboratory for OCB testing, and noted, despite the limited quality

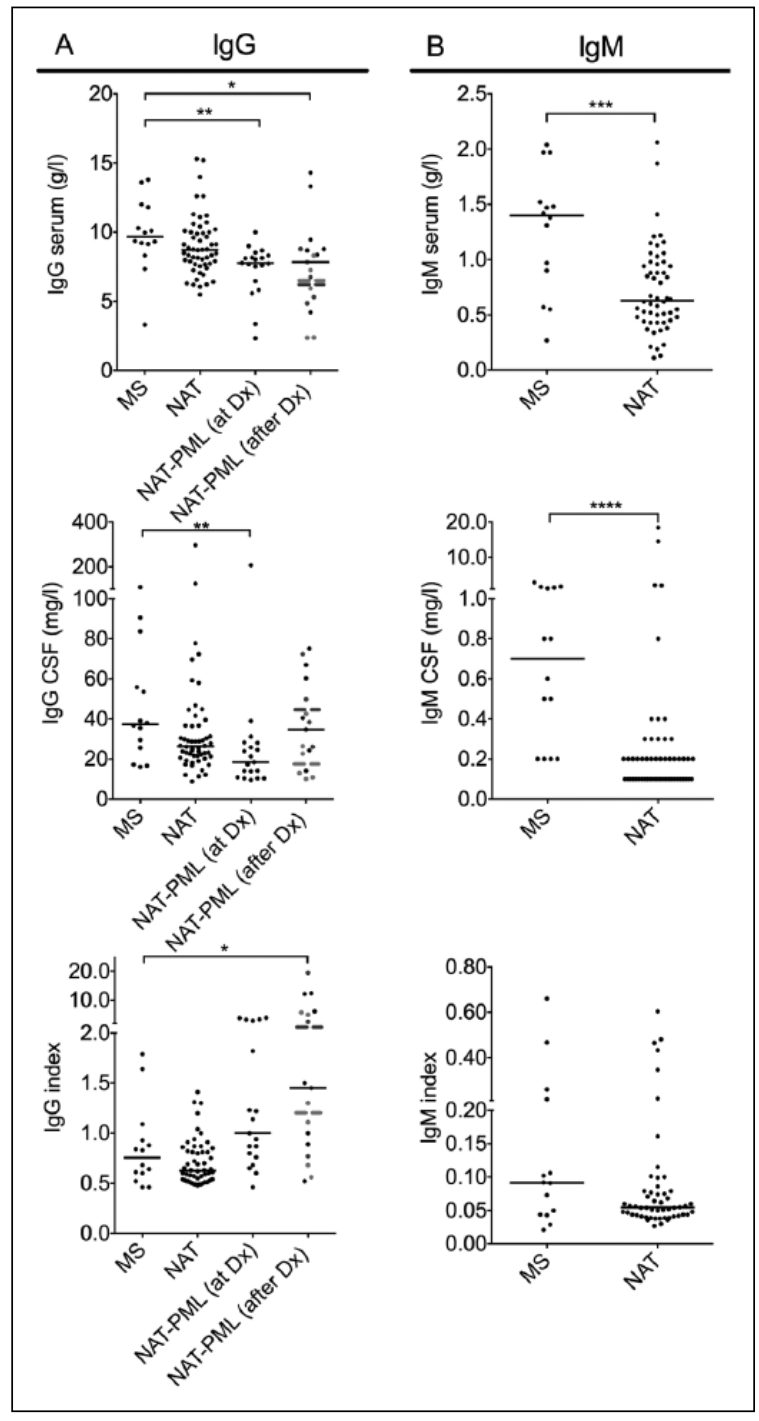

Figure 2. Levels of total IgG in blood and cerebrospinal fluid (CSF) and the IgG index (A), and levels of IgM in blood and CSF and the IgM index (B) are shown. Bars represent the median. Statistics: Kruskal-Wallis (A); Mann-Whitney (B). MS: patients with MS not treated with natalizumab; NAT: patients with MS treated with natalizumab; NAT-PML (at Dx): Patients with natalizumab-associated PML and sampling at time of first positive qPCR for JCV DNA in CSF; NAT-PML (after Dx): Patients with natalizumab-associated PML and with sampling available after diagnosis of PML; red dots mark samplings within 4, blue dots samplings after 4 weeks of start with plasma exchange; the interrupted red or blue bars indicate the corresponding median.

related to pre-analytic quality issues, a decline of the intensity of some of the bands during therapy. Such a decline in intensity might result in findings below the detection threshold of other laboratories, and the reporting of a negative OCB status. The observed decline in OCB intensity was in line with a noted 


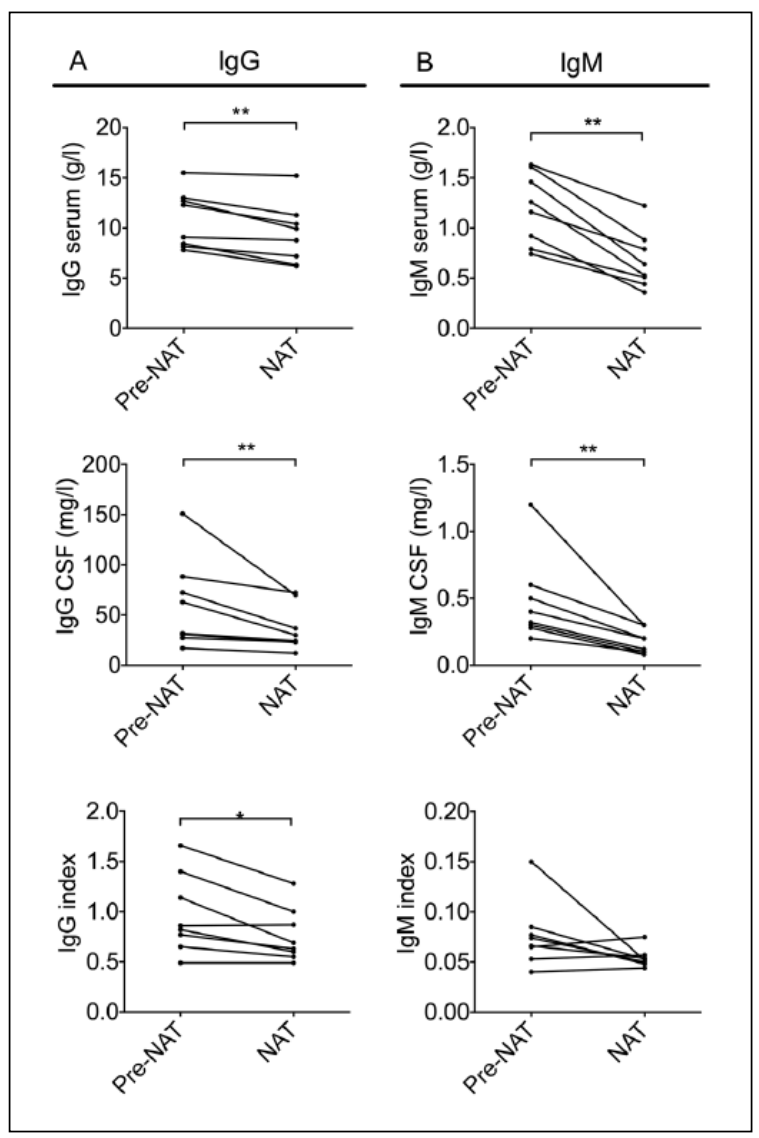

Figure 3. Levels of total $\mathrm{IgG}$ in blood and cerebrospinal fluid (CSF) and the IgG index (A), and levels of IgM in blood and CSF and the IgM index (B). Pre-NAT: prior to therapy with natalizumab; NAT: during therapy with natalizumab. Statistics: Wilcoxon.

decrease of the IgG index, as well as the absolute IgGloc concentration and the reduced polyspecific reactivity toward measles, rubella and zoster. In summary, our study in conjunction with previously published data is suggestive for a quantitative rather than a qualitative reduction of the intrathecally produced $\operatorname{IgG}$ fraction during therapy with natalizumab.

The majority of patients with untreated MS have an increased IgG index, OCBs in CSF, and a pathological antibody index toward measles, rubella, and zoster. ${ }^{22}$ Thus, the decline in all these parameters observed in our study during natalizumab treatment implicate complex, most likely indirect, effects on B-lymphocyte function. Natalizumab targets the $\alpha 4$-integrin, which is expressed on B and T cells in a tissue-specific manner. ${ }^{23}$ We did not mechanistically study how this mode of action may translate into a reduced peripheral and intrathecal antibody production. We assume that therapy-induced alterations of the B and T cell homoeostasis might represent a possible explanation for the changes observed. Within our current study, we noted a pronounced increase of the relative number of $\mathrm{B}$ cells in blood. Others have made similar observations. ${ }^{6,7}$ This might not be surprising, considering the fact that various $\alpha 4$-dependent pathways control B cell homeostasis. VLA-4 ( $\alpha 4 \beta 1)-$ VCAM-1 binding mediates homing of IgG-producing plasma cells to the bone marrow, and interacting of bone marrow stroma cells with developing B cells. Mature B cells use $\alpha 4 \beta 1$ to bind to VCAM- 1 on follicular dendritic cells in the B cell follicles of secondary lymphoid organs, including the spleen, lymph nodes, and Peyer's patches. The $\alpha 4 \beta 7-M A d C A M-1$ interaction is of importance for homing of naive and memory lymphocytes into the intestinal mucosa. ${ }^{24}$ Overall, blockade of the $\alpha 4$ integrin may directly impact B cell homing and adhesion to natural niches, which in turn might explain the increased numbers of B cells in the periphery, the loss of pro-survival stimuli in natural niches, ${ }^{25}$ and a reduced peripheral antibody production during natalizumab therapy. Similar processes might also control the homing, adhesion, and interactions of lymphocytes in ectopic meningeal follicles considered relevant to the production of Ig in CSF. ${ }^{26}$

Disturbances in the $\mathrm{B}$ and $\mathrm{T}$ cell interactions and antigen recognition may also contribute to lower Ig levels. We noted a relative reduction of $\mathrm{B}$ cells and an inversion of the $\mathrm{CD} 4 / \mathrm{CD} 8$ ratio in $\mathrm{CSF}$, whereas monocytes/macrophages - reported to display a more moderate decrease in the $\alpha 4$ and $\beta 1$ surface expression in response to natalizumab as compared with $\mathrm{B}$ and $T$ cells $^{27}$ - appeared to show a compensatory relative increase in CSF. Natalizumab is known to block transmigration of B cells, T cells, plasma cells, and antigen-presenting cells across the blood-CSF barrier. ${ }^{2,3}$ The altered cellular immune surveillance in the central nervous system (CNS), together with altered antigen recognition, and $\mathrm{B}$ and $\mathrm{T}$ cell interactions, could secondarily contribute to a quantitative reduction of intrathecally produced Ig.

Reported natalizumab-induced alteration of gene expression is another possible explanation for changes in Ig production. Genes involved in B cell differentiation might be re-regulated by natalizumab, ${ }^{28}$ a process in which epigenetic mechanisms could play a role. ${ }^{29}$ The observation of an increased number of hematopoetic progenitors, pre-B and B cells in peripheral blood of MS patients during therapy with natalizumab, along with the finding of the transcription factor Spi-B being up-regulated in response to natalizumab therapy in CD34+ and CD19+ cells, ${ }^{30}$ have been claimed to be relevant to pathogenesis of PML in patients with MS. In such a hypothesis, up-regulated 


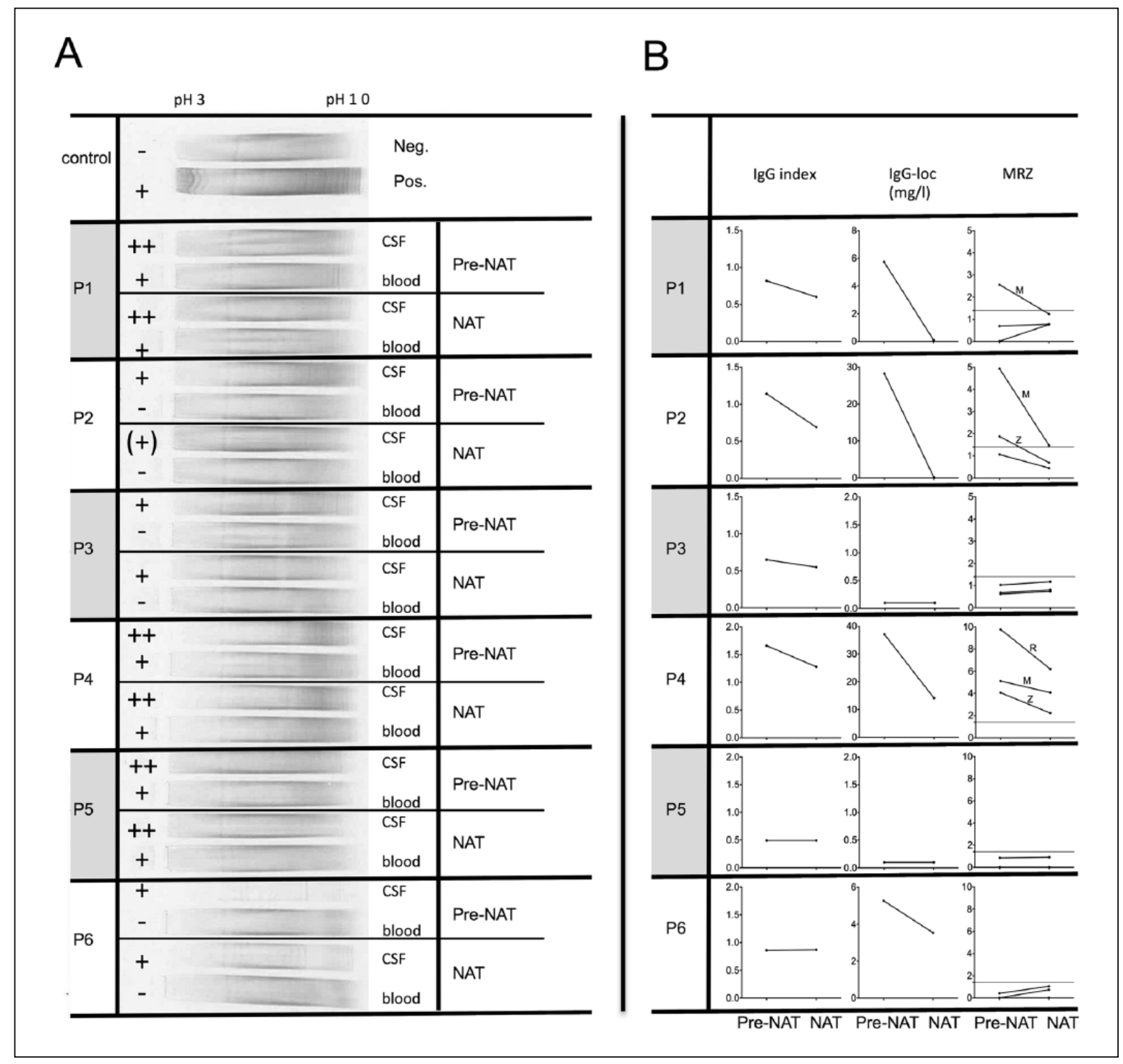

Figure 4. In six individuals (P1-P6) with samples pairs available before (Pre-NAT) and during natalizumab therapy (NAT) oligoclonal bands (OCBs) detected by isoelectric focusing on polyacrylamide gels are shown (A). In the same patients, the IgG index (left), the absolute IgG concentration locally produced in the central nervous system (IgG-loc, middle), and the antibody index to measles (M), rubella (R) and varizella zoster virus (Z, right) are depicted (B). Preanalytic sample handing (stored samples, two freezing-thawing cycles prior to testing), and scanning procedure affected the quality of the OCB plot shown.

Spi-B would promote JCV reactivation via interaction with the non-coding control region of latent JCV in $\mathrm{B}$ cells or pre-B cells. B cells that harbour reactivated JCV would then carry the virus to the brain and promote CNS infection. ${ }^{31}$ However, we previously failed to detect JCV DNA in peripheral blood mononuclear cells (PBMCs) or CD34+ cells of patients treated with natalizumab, ${ }^{32}$ although recently published data supports the view of B cells and/or CD34+ progenitor cells as a possible reservoir for JCV. ${ }^{33,34}$ However, it remains unclear how JCV-infected B cells, shown to have reduced expression of $\alpha 4 \beta 1$ during natalizumab therapy, ${ }^{35}$ and display impaired migration across endothelial barriers, ${ }^{8}$ would reach the CNS. Indeed, B cells were barely detectable in CSF of our natalizumab-treated patients. In addition, gene expression studies in PBMCs that report up-regulation of genes involved in $\mathrm{B}$ cell activation ${ }^{28}$ could possibly be biased by the pronounced increase of $B$ cells in peripheral blood and the changes in the composition of B cell subpopulations discussed above.

Limitations to our study are the cross-sectional design, the longer treatment duration of patients with 
natalizumab-associated PML compared with MS patients treated with natalizumab not developing PML, the lack of information on clinical disease activity scores throughout the study period (relapse rate, Expanded Disability Status Scale), and the fact that we did not assess antibody subclasses or specificity of antibodies. Nevertheless, our findings of lowered Ig levels in blood and CSF are consistent with the literature, and we are the first to show that patients with PML have also lowered overall IgG levels at time of diagnosis. In addition, we have previously observed that anti-JCV IgG antibody levels might decrease after treatment initiation with natalizumab. ${ }^{14}$ It is unclear, to date, if these changes may predispose to the development of PML. Indeed, high and increasing anti-JCV antibody levels have been observed in patients with natalizumab-associated PML at time of diagnosis of PML. ${ }^{14}$ This fits the widely accepted opinion that the humoral anti-JCV response alone might not be sufficient, while JCV-specific T-cell responses are considered key players in prevention of PML, and elimination of JCV in individuals with PML. ${ }^{12}$ Nonetheless, the causative pathogen JCV appears to undergo intra-individually acquired viralgenetic changes in the coding and non-coding region. ${ }^{36,37}$ During immunosuppression, the frequency of viral replication cycles at places of persistency, such as the renal tissue, might increase. This could be associated with a higher likelihood of the acquisition of pathogenic PML variants that eventually escape immune surveillance and cause PML. All arms of the adaptive immunity, including $B$ cells as antigen-presenting cells, B cells for interactions with T cells, and high-affinity maturation of antibodies in germinal centres may be required to limit viral turnover, to neutralize virus in the blood stream, and to control virus in the CNS compartment that either reaches it via the blood stream or becomes locally reactivated from latently infected CNS cells. ${ }^{38}$ Interestingly, we recently could show that the anti-JCV antibody response is strongly controlled by human leukocyte antigen class II variants. ${ }^{39}$ Recently presented data on genome-wide transcript profiling in patients with PML versus non-PML showed a downregulation of genes involved in the regulation of immunoglobulins and B cell activation. ${ }^{40}$ Overall, anti-JCV antibodies may not be sufficient to prevent PML, but B cells and their effector function in total might still play an important role in anti-JCV defence.

The post-PML samples of our current study show that during the course of PML following cessation of natalizumab and PLEX (most obvious in samples obtained after 4 weeks or later following PLEX initiation), total IgG levels in CSF appear to rise as well as the IgG index. This may be a surrogate of reconstitution of adaptive immune competence in the CNS. Accordingly, we have previously shown that a majority of patients with natalizumab-associated PML have already developed a strong intrathecal anti-JCV antibody response at time of diagnosis of PML, which later increases and may aid in the early recognition of this dreaded complication. ${ }^{18}$

The extent to which changes in cellular as well as humoral immune composition described in this study may account for the development of PML in patients during selective immunosuppression needs to be studied in greater detail in larger patient cohorts. A more detailed assessment of global B cell effector functions may aid to elucidate the still incompletely understood pathogenesis of natalizumab-associated PML.

\section{Conflict of interest statement}

CW: speakers honoraria, Bayer Healthcare; TD: travel expenses Novartis Pharma, Genzyme; AKM: travel expenses, Biogen Idec; RG: speaker's honoraria, grant support, board honoraria, Biogen Idec, Bayer, Teva, Novartis, Merck Serono; TK: travel expenses, personal compensation (speaking fees) Bayer Healthcare/Teva Pharma, Merck Serono, Novartis, Sanofi-Aventis/ Genzyme, Biogen Idec, grant support, Novartis Pharma; RH: board membership, consultancy, speaking fees, Novartis, Teva, Biogen Idec, Merck Serono, Bayer Schering, Sanofi-Aventis, Genzyme; grant support, Novartis, Teva, Biogen Idec, Merck Serono, Bayer Schering, Sanofi-Aventis; MM: speaker's honoraria, board honoraria Biogen Idec, Bayer, Teva, Novartis, Merck Serono, Genzyme, Sanofi-Aventis; MSta reports grants and personal fees from Biogen Idec, grants and personal fees from Novartis, grants and personal fees from Bayer Healthcare, grants and personal fees from Teva, personal fees from Sanofi-Aventis, personal fees from Baxter, personal fees from CSL Behring, personal fees from Grifols, outside the submitted work; TW: has received honoraria from Bayer, Biogen Idec, Genzyme, Novartis, Sanofi-Aventis for consulting and lecturing. As a member of the scientific advisory board of the PML consortium he is receiving honoraria for consulting; CK: speakers honoraria, consulting fees and grant support from Bayer Healthcare, Sanofi-Genzyme, Teva, Merck Serono, Boehringer Ingelheim, Biotronik, Biogen Idec, Novartis, CSL Behring, Pfizer, and Siemens; MPW: received honoraria for lectures and consultancy, Biogen Idec. AS: grant support, Biogen Idec; travel support, Biogen Idec, Genzyme/Sanofi, Merck Serono; lecture honoraria, Biogen Idec, Genzyme/Sanofi, Teva, Merck Serono; advisory board, Biogen Idec, Genzyme/Sanofi, Teva, Merck Serono; TO: grant support, personal fees, Biogen Idec, Novartis, 
Genzyme, Merck. HPH: has received honoraria for consulting and speaking at symposia from Bayer, Biogen Idec, Genzyme, Merck Serono, Novartis Pharma, Roche and Teva Sanofi-Aventis, with approval by the Rector of Heinrich-Heine-University. HT: has received honoraria for consulting and lecturing, travel expenses for participating at symposia, and financial support for research from Bayer, Biogen Idec, Genzyme, Merck, Novartis, Roche Diagnostics, Siemens Diagnostics and TEVA. OA: personal fees, Biogen Idec. BCK: has received honoraria for lecturing, travel expenses for attending meetings, and financial support for research from Bayer Health Care, Biogen Idec, Genzyme/Sanofi-Aventis, Grifols, Merck Serono, Mitsubishi Europe, Novartis, and TEVA. MSte, VLeh, GvG, VS, VLim and DH have nothing to disclose.

\section{Funding}

This work was supported by a local faculty grant (Research Commission of Heinrich Heine University, Duesseldorf [HHU]; OA) and a grant from the German Ministry for Education and Research (BMBF; German Competence Network MS [KKNMS], Natalizumab Pharmacovigilance Study, 01GI1002; BCK.). CW was supported by a European Committee for Treatment and Research in MS fellowship stipend. The research leading to these results received support from the Innovative Medicines Initiative Joint Undertaking under grant agreement No. 115303, resources of which are composed of a financial contribution from the European Union's Seventh

Framework Program (FP7=2007-2013) and European Federation of Pharmaceutical Industries and Associations companies' in-kind contribution (to HPH, BCK, CW). The MS Center at the Department of Neurology, HHU is supported by the Walter and Ilse Rose Foundation. RH was supported by the German Research Foundation (DFG CRC TRR128, Z2) and BMBF (KKNMS). MSta is supported by the NiedersachsenResearchNetwork onNeuroinfectiology of the Ministry of Science and Culture of Lower Saxony and the BMBF (KKNMS). We highly appreciate the excellent technical support by Tatjana Males.

\section{References}

1. Yednock TA, Cannon C, Fritz LC, et al. Prevention of experimental autoimmune encephalomyelitis by antibodies against alpha 4 beta 1 integrin. Nature 1992; 356: 63-66.

2. del Pilar Martin M, Cravens PD, et al. Decrease in the numbers of dendritic cells and CD4+ T cells in cerebral perivascular spaces due to natalizumab. Arch Neurol 2008; 65: 1596-1603.
3. Stuve O, Marra CM, Jerome KR, et al. Immune surveillance in multiple sclerosis patients treated with natalizumab. Ann Neurol 2006; 59: 743-747.

4. Warnke C, Mausberg AK, Stettner M, et al. Natalizumab affects the T-cell receptor repertoire in patients with multiple sclerosis. Neurology 2013; 81: 1400-1408.

5. Zohren F, Toutzaris D, Klarner V, et al. The monoclonal anti-VLA-4 antibody natalizumab mobilizes CD34+ hematopoietic progenitor cells in humans. Blood 2008; 111: 3893-3895.

6. Krumbholz M, Meinl I, Kumpfel T, et al. Natalizumab disproportionately increases circulating pre-B and B cells in multiple sclerosis. Neurology 2008; 71: 1350-1354.

7. Planas R, Jelcic I, Schippling S, et al. Natalizumab treatment perturbs memory- and marginal zonelike B-cell homing in secondary lymphoid organs in multiple sclerosis. Eur J Immunol 2012; 42: 790-798.

8. Saure C, Warnke C, Zohren F, et al. Natalizumab and impedance of the homing of CD34+ hematopoietic progenitors. Arch Neurol 2011; 68: 1428-1431.

9. Jing D, Oelschlaegel U, Ordemann R, et al. CD49d blockade by natalizumab in patients with multiple sclerosis affects steady-state hematopoiesis and mobilizes progenitors with a distinct phenotype and function. Bone Marrow Transplant 2010; 45: 1489-1496.

10. Koopman G, Keehnen RM and Pals ST. Interaction through the LFA-1/ICAM-1 pathway prevents programmed cell death of germinal center B cells. Adv Exp Med Biol 1993; 329: 387-392.

11. Bloomgren G, Richman S, Hotermans C, et al. Risk of natalizumab-associated progressive multifocal leukoencephalopathy. N Engl J Med 2012; 366: 1870-1880.

12. Warnke C, Menge T, Hartung HP, et al. Natalizumab and progressive multifocal leukoencephalopathy: What are the causal factors and can it be avoided? Arch Neurol 2010; 67: 923-930.

13. Fine AJ, Sorbello A, Kortepeter C, et al. Central nervous system herpes simplex and varicella zoster virus infections in natalizumab-treated patients. Clin Infect Dis 2013; 57: 849-852.

14. Warnke C, Ramanujam R, Plavina T, et al. Changes to anti-JCV antibody levels in a Swedish national MS cohort. J Neurol Neurosurg Psychiatry 2013; 84: 1199-1205.

15. Selter RC, Biberacher V, Grummel V, et al. Natalizumab treatment decreases serum IgM and IgG levels in multiple sclerosis patients. Mult Scler 2013; 19: 1454-1461. 
16. Mancuso R, Franciotta D, Rovaris M, et al. Effects of natalizumab on oligoclonal bands in the cerebrospinal fluid of multiple sclerosis patients: A longitudinal study. Mult Scler 2014; In press.

17. Harrer A, Tumani H, Niendorf S, et al. Cerebrospinal fluid parameters of B cell-related activity in patients with active disease during natalizumab therapy. Mult Scler 2013; 19: 1209-1212.

18. Warnke C, von Geldern G, Markwerth P, et al. The CSF JCV antibody index for diagnosis of natalizumab-associated PML. Ann Neurol 2014; In press.

19. Keir G, Luxton RW and Thompson EJ. Isoelectric focusing of cerebrospinal fluid immunoglobulin $\mathrm{G}$ : An annotated update. Ann Clin Biochem 1990; 27: 436-443.

20. Andersson M, Alvarez-Cermeno J, Bernardi G, et al. Cerebrospinal fluid in the diagnosis of multiple sclerosis: A consensus report. J Neurol Neurosurg Psychiatry 1994; 57: 897-902.

21. Reiber $\mathrm{H}$ and Peter JB. Cerebrospinal fluid analysis: Disease-related data patterns and evaluation programs. J Neurol Sci 2001; 184: 101-122.

22. Reiber H, Ungefehr S and Jacobi C. The intrathecal, polyspecific and oligoclonal immune response in multiple sclerosis. Mult Scler 1998; 4: 111-117.

23. Leite RP, Carmo-Fonseca M, Cabecadas J, et al. Differential expression of cell adhesion molecules in the functional compartments of lymph nodes and tonsils. Clin Mol Pathol 1995; 48: M93-M100.

24. von Andrian UH and Engelhardt B. Alpha4 integrins as therapeutic targets in autoimmune disease. $N$ Engl $J$ Med 2003; 348: 68-72.

25. Koopman G, Keehnen RM, Lindhout E, et al. Adhesion through the LFA-1 (CD11a/CD18)ICAM-1 (CD54) and the VLA-4 (CD49d)-VCAM-1 (CD106) pathways prevents apoptosis of germinal center B cells. J Immunol 1994; 152: 3760-3767.

26. Serafini B, Rosicarelli B, Magliozzi R, et al. Detection of ectopic B-cell follicles with germinal centers in the meninges of patients with secondary progressive multiple sclerosis. Brain Pathol 2004; 14: $164-174$.

27. Harrer A, Wipfler P, Einhaeupl M, et al. Natalizumab therapy decreases surface expression of both VLA-heterodimer subunits on peripheral blood mononuclear cells. J Neuroimmunol 2011; 234: $148-154$.

Visit SAGE journals online

(S) SAGE journals of blood cell subpopulations of multiple sclerosis patients. J Neuroimmunol 2008; 194: 153-164.

29. Meira M, Sievers C, Hoffmann F, et al. MiR-126: A novel route for natalizumab action? Mult Scler 2014; 20: $1363-1370$.

30. Marshall LJ, Ferenczy MW, Daley EL, et al. Lymphocyte gene expression and JC virus noncoding control region sequences are linked with the risk of progressive multifocal leukoencephalopathy. J Virol 2014; 88: 5177-5183.

31. Houff SA, Berger J, Major EO. Response to Linberg, et al. Natalizumab alters transcriptional expression profiles of blood cell subpopulations of multiple sclerosis patients. J Neuroimmunol 2008; 204: 155-156; author reply 157.

32. Warnke C, Smolianov V, Dehmel T, et al. CD34+ progenitor cells mobilized by natalizumab are not a relevant reservoir for JC virus. Mult Scler 2011; 17: $151-156$.

33. Frohman EM, Monaco MC, Remington G, et al. JC virus in CD34+ and CD19+ cells in patients with multiple sclerosis treated with natalizumab. JAMA Neurol 2014; 71: 596-602.

34. Chalkias S, Dang X, Bord E, et al. JC virus reactivation during prolonged natalizumab monotherapy for multiple sclerosis. Ann Neurol 2014; 75: 925-934.

35. Skarica M, Eckstein C, Whartenby KA, et al. Novel mechanisms of immune modulation of natalizumab in multiple sclerosis patients. J Neuroimmunol 2011; 235: $70-76$.

36. Reid CE, Li H, Sur G, et al. Sequencing and analysis of JC virus DNA from natalizumab-treated PML patients. J Infect Dis 2011; 204: 237-244.

37. Gorelik L, Reid C, Testa M, et al. Progressive multifocal leukoencephalopathy (PML) development is associated with mutations in JC virus capsid protein VP1 that change its receptor specificity. J Infect Dis 2011; 204: 103-114.

38. Bayliss J, Karasoulos T and McLean CA. Frequency and large T (LT) sequence of JC polyomavirus DNA in oligodendrocytes, astrocytes and granular cells in non-PML brain. Brain Pathol 2012; 22: 329-336.

39. Sundqvist E, Buck D, Warnke C, et al. JC polyomavirus infection is strongly controlled by human leucocyte antigen class II variants. PLoS Pathog 2014; 10: e1004084.

40. Plavina T, Carulli J, Li H, et al. B cell-specific signal in whole blood and serum of natalizumab-treated PML patients is revealed using transcript and protein profiling. Neurology 2014; 82: Supplement S31.004. 